Recherches en didactique des langues et des cultures

Les cahiers de l'Acedle

$3 \mid 2008$

Recherches en didactique des langues

\title{
La dimension émotionnelle de la communication en situation interculturelle
}

L'expression non verbale de la colère chez des locuteurs anglo-

australiens, français et italiens

Colette Mrowa-Hopkins et Antonella Strambi

\section{OpenEdition}

1 Journals

Édition électronique

URL : http://journals.openedition.org/rdlc/2891

DOI : 10.4000/rdlc.2891

ISSN : 1958-5772

Éditeur

ACEDLE

Référence électronique

Colette Mrowa-Hopkins et Antonella Strambi, « La dimension émotionnelle de la communication en situation interculturelle », Recherches en didactique des langues et des cultures [En ligne], 3 | 2008, mis en ligne le 30 juillet 2008, consulté le 02 mai 2019. URL : http://journals.openedition.org/rdlc/2891 ; DOI : $10.4000 /$ rdlc. 2891

Ce document a été généré automatiquement le 2 mai 2019.

\section{c) (7) $(-)$}

Recherches en didactique des langues et des cultures is licensed under a Creative Commons AttributionNonCommercial-NoDerivatives 4.0 International License 


\section{La dimension émotionnelle de la communication en situation interculturelle}

L'expression non verbale de la colère chez des locuteurs angloaustraliens, français et italiens

Colette Mrowa-Hopkins et Antonella Strambi

\section{Introduction}

1 Notre étude s'inscrit dans une perspective à la fois interrelationnelle et interculturelle et porte sur la dimension émotionnelle de la communication entre locuteurs de groupes culturellement différenciés. La démarche que nous avons adoptée s'articule autour d'une réflexion théorique plus large portant sur le comportement non verbal dans l'expression et la communication des émotions dans les relations interpersonnelles impliquant divers degrés d'investissement de soi et de distanciation.

2 L'expression et la communication des émotions à travers le comportement non verbal a fait l'objet d'un certain nombre d'analyses théoriques proposant un ensemble de normes d'interaction et de compréhension qui régissent les échanges entre partenaires conversationnels ${ }^{1}$. Le point de départ de notre réflexion s'appuie sur la constatation que ces normes, ou principes prescriptifs auxquels se soumettent collectivement les membres d'une culture donnée (Gudykunst \& Ting-Toomey, 1988: 61), relèvent de l'implicite social et culturel. Il apparaît que le non-respect ou la méconnaissance de ces principes génère le plus souvent des comportements en porte-à-faux et des malentendus pouvant engendrer de véritables conflits, les locuteurs natifs ayant tendance à prêter aux locuteurs étrangers, particulièrement si ceux-ci parlent couramment leur langue, des intentions plus ou moins malveillantes (Gass \& Varonis, 1991). Il en résulte des jugements défavorables vis-à-vis du groupe culturel considéré, renforçant ainsi les croyances les plus communément répandues à leur propos, et de nature le plus souvent stéréotypée (Béal, 1990 ; 1992). 
3 Il est généralement admis que ces normes culturelles opèrent à un niveau subconscient (Andersen \& Guerrero, 1998; Peeters, 2003) et qu'elles sont acquises tacitement par les locuteurs natifs, d'où la notion implicite qu'elles deviennent automatiquement accessibles aux apprenants de langue étrangère lorsqu'ils atteignent un niveau avancé de compétence linguistique. Or, les recherches en didactique des langues secondes ou étrangères montrent que sans une réflexion personnelle ou un apprentissage spécifique cette prise de conscience interculturelle est rare (Porter \& Samovar, 1998 ; Kasper, 1997). La nécessité d'expliciter ces normes d'interaction et de compréhension nous parait donc essentielle afin de fournir aux apprenants l'occasion de maitriser pleinement la langue et la culture de l'autre.

4 Les modalités culturelles d'investissement de soi et la prise en compte de la dimension émotionnelle dans le discours, de même que les aspects socio-pragmatiques de la communication en général, sont pratiquement ignorées des méthodes d'apprentissage de langues (Liddicoat, 1997). Ceci est dû au fait qu'elles relèvent essentiellement d'activités discursives, et qu'elles ne peuvent donc être saisies en termes de compétences spécifiques (Vion, 1992). C'est pour cette raison que nous préconisons une démarche pédagogique basée sur l'intégration de ressources qui prennent appui aussi bien sur des faits observables que sur des analyses théoriques. Avant d'en venir à cet aspect plus proprement didactique, nous aimerions brièvement passer en revue les études les plus récentes qui ont orienté notre recherche sur le comportement non verbal dans la communication émotionnelle en situation interculturelle. Notre attention se portera en particulier sur les manifestations de la colère, et les différences observées entre les trois groupes culturels sélectionnés dans notre étude.

\section{Le point de vue de la recherche}

$5 \quad$ Il est clair que les signes para-verbaux et non verbaux jouent un rôle prépondérant dans toute communication humaine. Les expressions du visage, les inflexions de la voix, les gestes et autres mouvements corporels, sans oublier la façon de se maintenir ou de s'habiller, sont des indices particulièrement révélateurs de la personnalité, des croyances et des valeurs, ainsi que du statut social des locuteurs en présence aux cours de leurs échanges conversationnels (Birdwhistell, 1970 ; Burgeon et al, 1989; Mehrabian, 1969, 1972). En outre, des chercheurs comme Ekman et Freisen (1969) aux Etats-Unis, Cosnier et Brossard (1984) en France, ont identifié les comportements non verbaux comme indices principalement susceptibles de signaler des changements dans les relations interpersonnelles, et comme source de toute évaluation, opinion et jugement que forment les individus sur leurs interlocuteurs.

6 La plupart des recherches plus récentes qui se concentrent sur l'expression des émotions dans une perspective interculturelle s'accordent sur la définition que proposent Markus et Kitayama (1994), à savoir que les émotions sont « un ensemble de scripts partagés socialement et composés de divers processus - physiologiques, subjectifs, et comportementaux » (notre traduction). Cette définition présente un double avantage pour notre étude, d'une part, celui de rendre l'expression des émotions plus accessible à l'observation, et d'autre part, celui de suggérer que différents groupes sociaux vont anticiper diversement les circonstances plus propices à l'expression d'une émotion en réponse à une situation contextuelle particulière. Ce comportement expressif implique, en d'autres termes, que bien que l'expérience des émotions apparaisse comme un invariant 
culturel, la façon dont les émotions se manifestent, ou alternativement sont contrôlées, négociées, etc., sont également spécifiques parce qu'elles renvoient à des représentations et à des attentes en matière de comportements qui sont partagées par une collectivité sociale et culturelle.

Un petit nombre d'émotions, telles que la joie, la crainte, la honte, la colère, ont été identifiées comme faisant partie d'un stock d'invariants universels. Parmi celles-ci, la colère nous semble particulièrement intéressante parce qu'elle est une des émotions les plus « actives » selon Matsumoto, Wallbott et Scherer (1994: 237) - c'est-à-dire que son expression s'accompagne d'un déploiement de signes para-verbaux et non verbaux faisant intervenir à la fois le visage, la voix et les mouvements du corps. Ces auteurs ont également souligné que la colère, de même que la joie, fait partie des émotions les plus « sociabilisées " (1994 : 237), et que ces deux émotions sont aussi très extériorisées - à savoir, que les réactions expressives de la colère dépassent ses manifestations physiologiques. De plus, la colère est considérée comme une émotion "négative " (Matsumoto, Kudoh, Scherer \& Wallbott, 1988). Cette dernière caractéristique implique que si elle fait partie des émotions les plus fréquemment ressenties, son degré d'expressivité dans un contexte social est beaucoup moins fort (Matsumoto, Wallbott et Scherer, 1994) parce que les règles de la vie sociale ${ }^{2}$ prescrivent la retenue et découragent le déploiement de la colère dans sa pleine intensité. Etant donné la variété des normes socioculturelles, la colère nous semble particulièrement exploitable parce qu'il est plus que probable que différents groupes culturels varient leurs modalités de contrôle selon le degré de manifestation émotionnelle jugé acceptable dans la culture d'origine.

\section{Différences observées entre les groupes anglo- australien, français et italien}

8 Les trois groupes culturels sélectionnés dans notre étude présentent des différences intéressantes sur le plan d'un certain nombre de dimensions socioculturelles, ce qui laisse anticiper des différences dans la façon dont le comportement émotionnel se déploie à travers des indices non verbaux.

Certaines de ces différences ont déjà fait l'objet de recherches précédentes. Par exemple, dans son étude sur les interactions entre Anglo-australiens et Français sur leurs lieux de travail, Béal (1990 : 28) note que le comportement émotif est plus souvent manifeste dans la culture française, et ajoute que « la colère, en particulier, est beaucoup plus acceptable socialement que dans la culture australienne, ce qui peut générer des conflits. » (notre traduction). Béal (1992 : 25) a aussi observé que les participants australiens ont tendance à décrire leurs collègues français comme "préoccupés d'eux-mêmes, impérieux et manquant d'égards envers les autres"(notre traduction) tandis que les Français considèrent leurs collègues australiens comme indifférents et manquant de sincérité. Ces différentes perceptions s'expliquent en partie par un déséquilibre dans les contenus informatifs échangés au cours de conversations informelles ainsi que dans les différentes modalités d'investissement des locuteurs individuels.

Ces différences observables pourraient également s'expliquer par un degré plus ou moins fort d'adhésion ou de distanciation par rapport aux notions de pouvoir hiérarchique et de distance sociale communiquées par la langue française. Etant donné que les rapports hiérarchiques sont clairement établis à travers des moyens linguistiques tels que le 
tutoiement et le vouvoiement, l'investissement de ses propres sentiments ou émotions dans l'échange n'implique pas nécessairement une plus grande intimité et ne représente pas forcément un indice de rapprochement social. Par contre, une réduction du degré d'investissement personnel permet aux Australiens de maintenir une certaine distance sociale, qui par ailleurs indique le respect du domaine privé de la personne.

De plus, Béal introduit l'idée que les Français éprouvent le besoin de s'exprimer et "d'avoir une opinion sur tout sujet quel qu'il soit», et selon un des participants "faire savoir aux autres ce que l'on pense peut être une forme de respect» (1992: 47). Au contraire, la "gentillesse affable $\|^{3}$ est une des valeurs fondamentales de la culture australienne qui se traduit par l'effort d'entretenir une relation informelle et le besoin de préserver une certaine harmonie dans les rapports sociaux. Il s'ensuit qu'afin d'éviter des conflit potentiels, le maintien d'une certaine distance sociale est souvent préférable à la confrontation ouverte des idées.

Des observations similaires ont été faites par rapport aux normes socioculturelles italiennes. Par exemple, Carroli, Pavone et Tudini (2003 : 184) notent que « dans la culture italienne, le conflit est généralement considéré comme un phénomène naturel, un moyen même agréable et créatif permettant de déboucher sur la voie du consensus ». La présence de conflit dans l'échange conversationnel est pour une large part influencée par la distance relationnelle entre les interlocuteurs, leur style individuel, et leurs habitudes culturelles (Bazanella, 1994). En outre, les Anglo-australiens interprètent souvent la vivacité du ton, la force de la tonalité, l'expressivité gestuelle et l'animation du visage observées au cours de conversations italiennes comme les signes d'une argumentation violente. Vice et versa, la restreinte dans l'expression démontrée par les sujets Angloaustraliens est souvent interprétée comme de la froideur par les cultures méditerranéennes. Wierzbicka (1999:19) remarque, par exemple, que le terme anglais emotional, correspondant au français "émotif " comporte des connotations négatives, alors qu'en Italien, « le terme emozione a une interprétation plus proche de la passion. »

Compte tenu des observations précédentes, il est bien probable que les Français et les Italiens auront tendance à déployer un comportement d'émotivité plus négatif dans leurs rapports interpersonnels avec des partenaires de culture anglo-saxonne étant donné que, pour ceux-ci, la manifestation publique d'émotions négatives va socialement à l'encontre de l'effort qu'ils font pour éviter tout conflit ou embarras. Les informations véritablement scientifiques relatives aux normes socioculturelles sont cependant rares et plusieurs chercheurs insistent sur le besoin urgent de la recherche d'établir une base de données reposant sur des observations et analyses de comportements en milieu authentique. C'est dans cette perspective que se situe notre étude dont l'objectif est de contribuer à une meilleure compréhension des normes socioculturelles réglant le comportement non verbal dans la communication émotionnelle, avec un éclairage particulier sur l'expression de la colère parmi les trois groupes culturels sélectionnés : anglo-australien, français et italien.

14 Avant de poursuivre, il convient d'expliquer les procédures que nous avons utilisées et appliquées pour obtenir des données susceptibles de constituer un corpus d'interactions en rapport avec les objectifs de notre étude. 


\section{Collecte de données}

15 Afin d'établir un corpus d'interactions pour l'étape préliminaire de notre étude, nous avons sélectionné des scènes de films contemporains dans lesquels nous étions certaines de trouver des manifestations de colère. En particulier, nous avons concentré notre recherche sur des scènes mettant en rapport des collègues de travail, des connaissances ou des étrangers. De ce fait, en excluant les scènes de rapport familiaux et d'amis proches, nous espérions trouver des normes d'interaction plus rigoureuses. De plus, nous pouvions nous attendre à ce que les risques encourus, à savoir les malentendus et la perception négative résultant de conduites jugées inappropriées par les locuteurs natifs, aient des répercussions plus sévères dans le contexte des relations de travail que dans celui des rapports entre intimes.

16 Le choix de films comme source de données peut s'expliquer par diverses raisons. D'abord, de par leur tissu narratif condensé, les films offrent une perspective privilégiée sur une variété de situations interpersonnelles qui ne pourraient être accessibles autrement. Ensuite, l'utilisation de films permet aux chercheurs de circonvenir les restrictions en place, principalement d'ordre éthique, dans la poursuite de recherches impliquant des sujets humains.

Quant à la validation scientifique, le choix de films comme source de données peut sans doute paraître discutable. Une des critiques le plus souvent avancée est celle du manque d'authenticité, étant donné que les films présentent des scénarios fictifs, et que le comportement d'un acteur n'est jamais tout à fait le même qu'un comportement spontané. Toutefois cet argument peut également s'appliquer à d'autres instruments de collecte dont se servent en particulier les chercheurs en ethnographie lorsqu'ils tentent de saisir les comportement des participants dans des milieux soi-disant "naturels"; l'observation est presque toujours une intrusion en dépit des précautions prises, et le risque de gaucherie de ceux qui se sentent regardés influence inévitablement les participants (Archer \& Akert, 1984 ; Scherer \& Ekman, 1982). Nous avons donc sélectionné des films qui, selon nous, présentaient des situations plausibles par rapport à notre expérience d'enseignantes et de chercheuses française et italienne, exerçant en Australie et ayant des contacts fréquents avec des Australiens d'origine anglo-saxonne, le groupe culturel dominant. Dans notre sélection, nous avons tenu compte de l'usage délibéré de la musique et des techniques spécifiques au tournage, angles de prises de vues et effets spéciaux, qui influencent à des degrés divers l'interprétation dramatique des événements narratifs dans le but de susciter l'engagement émotionnel des spectateurs.

L'autre dimension qui peut paraître problématique concerne la pseudo authenticité du comportement observé dans la mesure où le jeu de l'acteur peut influencer l'intensité de l'expression émotionnelle. Toutefois, selon Ekman (1986: 74), les «émotions posées " présentent néanmoins un avantage pour la recherche, à savoir qu'elles sont plus faciles à analyser ou à "répertorier" puisque leur expression tend à être mise en scène délibérément. En outre, les enregistrements vidéos offrent un avantage considérable aux chercheurs parce qu'ils leur donnent la possibilité d'étudier des dispositifs complexes de comportements en plus grand détail, en utilisant, par exemple, le retour en arrière, l'arrêt sur image, l'analyse plan par plan et le ralenti (Scherer \& Ekman, 1982). 


\section{Les critères de sélection des films}

19 Pour répondre aux objectifs que nous nous étions fixés dans cette étude, nous avons établi un corpus comprenant six films pour chaque pays (voir annexe 1), sortis sur les écrans au cours des dix dernières années (1993-2002), en nous basant sur les critères suivants :

- L'accessibilité des films en Australie, tout simplement pour des raisons pratiques ;

- La diversité de contextes situationnels présentés (réunions de famille, rencontres au bureau, classes ou conférences, etc.) ;

- La présentation des personnages en présence fréquente avec d'autres interactants, excluant de ce fait tout forme de communication à distance ;

- La représentation de personnages dont la conduite était conforme aux conventions morales et sociales acceptées par les individus de chaque groupe culturel. Autrement dit, il convenait que le comportement non verbal des acteurs n'apparaisse pas trop exagéré dans un but expressif ou comique voulu par le réalisateur. Ce critère est sans doute subjectif ; à cet égard, nous nous sommes fiés à notre propre compétence culturelle - en tant qu'individus appartenant au même groupe culturel que les personnages des films ;

- L'actualité des contextes situationnels - c'est-à-dire que nous avons écarté tous les films historiques ou d'époque dans le but de ne pas faire intervenir des variables associées aux changements trop évidents de comportements sociaux qui ont eu lieu au cours du siècle dernier, préférant nous concentrer sur les 10 à 15 dernières années.

\section{Analyse}

Étant donné que notre but était d'identifier les différents degrés d'expression non verbale de la colère, et surtout les cas où les règles en usage sont le plus susceptible de restreindre l'expression manifeste de la colère, nous avons exclu comme point de départ les changements de la voix et les expressions du visage. Ceci pour la simple raison qu'il peut exister des situations dans lesquelles la colère est anticipée mais les personnages contrôlent la manifestation explicite de leur colère conformément aux règles culturelles ou conventions sociales en usage. Il était donc nécessaire de sélectionner pour notre corpus des scènes qui révèlent l'expérience des émotions plutôt que leur manifestation apparente. Nous avons donc eu recours à une liste d'antécédents qui d'après une étude coordonnée par Scherer et Wallbott (1994) et conduite dans 37 pays sur un échantillon d'environ 3000 personnes, indique les motifs les plus courants de la colère.

Cette liste comprend 22 facteurs parmi lesquels :

- l'incapacité des autres à respecter les normes socioculturelles, à montrer du respect vis-à-vis des autres et de leur biens,

- les problèmes résultants de la circulation routière,

- la douleur physique,

- la séparation d'êtres chers,

- la non réalisation d'un but,

- l'injustice,

- le désagrément qui, par exemple, résulte de machines en panne.

Cette liste nous a permis de sélectionner des situations comparables qui pouvaient être identifiées comme sources potentielles engendrant colère. Une fois la sélection faite nous 
avons numérisé ces scènes et nous les avons sauvegardées sur DVD pour en garantir la facilité d'accès. Nous avons ensuite procédé à des analyses à la fois quantitatives et qualitatives (voir annexes 2 et $3^{4}$ ) en vue d'indiquer et d'évaluer l'information relative : (1) au type d'antécédent, (2) à la dimension de pouvoir et de distance interpersonnelle, et (3) au degré de déploiement explicite jugé d'après :

- le déplacement du corps (éloignement ou rapprochement vers une personne ou un objet) ;

- le comportement non verbal incluant le rire ou sourire, les pleurs ou sanglots et autres expressions du visage, les changements de la voix, les gestes, etc.;

- l'activité paralinguistique tels que les changements d'intonation ou de rythme du débit verbal, etc.

entendre de façon assez bruyante, ce qui va à l'encontre de l'image stéréotypée du groupe en comparaison des groupes italiens et français. Dans le film australien The Dish, par exemple, une scène montre une confrontation entre un employé d'une station satellite et son patron américain. L'employé devient de plus en plus énervé devant la supériorité arrogante du patron et ce qu'il interprète comme son manque de respect vis-à-vis du travail des employés. Il vocifère une pelletée d'insultes faisant appel à un répertoire d'images typiquement australiennes, ce qui lui permet d'affirmer son image identitaire face à «l'étranger » américain. Mais bien que la force perlocutoire de l'insulte soit ainsi atténuée par les usages métaphoriques du langage, sa démonstration de colère ne prête à aucune équivoque. Dans une autre scène très brève du même film, le patron australien réprimande gravement son employé en l'accusant d'un manque de compétence. A ce moment le patron s'avance vers l'employé et pointe un index accusateur, le menaçant du regard tout en haussant le ton de sa voix en signe de colère.

Dans d'autres cas, il nous semble que certains comportements qui peuvent paraître acceptables en France ou en Italie sont considérés hors normes dans un contexte australien. Par exemple, dans une scène du film Grégoire Moulin contre l'humanitéle protagoniste qui se trouve à la poste pour faire une photocopie fait face à un barrage de visages renfrognés de clients faisant la queue lorsqu'il leur demande de la monnaie. La colère manifestée par certains résulte d'une interprétation erronée qui est cependant tout à fait plausible dans le contexte socio-économique des grandes villes européennes où 
les gens sont sans cesse harcelés par des chômeurs et des sans-abris. Dans une autre scène, extraite du film italien Tre storie, une femme s'en prend au jardinier employé par un centre de réadaptation pour drogués parce qu'il a accidentellement coupé des fleurs auxquelles elle tenait. Son explosion de colère vise principalement le jardinier dont la faute, selon elle, est la conséquence directe de son handicap social. Il est presque certain que ce type de comportement discriminatoire ne serait pas exprimé si ouvertement dans un contexte australien.

Des analyses cadre par cadre des mouvements du visage ainsi que des spectrogrammes de la voix nous aideront à discerner dans une étape ultérieure des différences qui ne sont pas immédiatement apparentes. Nous espérons poursuivre cette recherche en collectant des données en situations authentiques. Ce qui nous semble particulièrement intéressant est d'analyser les perceptions que les locuteurs natifs italiens, français et angloaustraliens acquièrent à propos du caractère des personnages. Il est en effet possible que nos premiers résultats soient influencés par la source des données, et en particulier par les genres de fiction et les types de rapports interpersonnels dépeints au cinéma et surtout dans les films que nous avons sélectionnés. De plus, il est fort probable que des variables appartenant aux micro cultures, telles que des différences générationnelles, régionales, professionnelles, sexuelles, ou de niveaux socio-économiques, influencent aussi le degré d'intensité de l'expression émotionnelle. Toutefois, comme nos observations concordent avec celles des recherches précédentes, il ne fait pas de doute que notre corpus répond au besoin minimal de représentativité des comportements observables parmi les groupes culturels considérés.

Essentiellement, en regard de l'objectif secondaire que nous nous sommes fixé, ce projet nous a fait prendre conscience du potentiel qu'une telle analyse peut offrir dans une perspective pédagogique. Dans la dernière partie de cet article nous discutons de la pertinence de notre étude par rapport à une telle approche et nous suggérons quelques façons de l'intégrer dans des classes de langues, dont l'un des objectifs actuels d'apprentissage est le développement de la compétence interculturelle chez l'apprenant.

\section{Implications pour une démarche d'apprentissage interculturel}

D’après la définition élaborée par Byram (1997) la compétence interculturelle implique l'acquisition d'un ensemble de compétences à la fois cognitives, affectives et sociales, résumées succinctement par Barraja-Rohan (1999: 143) comme «l'ouverture sur la diversité culturelle et l'aptitude à reconnaître et à accepter les différences et à les intégrer de façon satisfaisante dans le temps réel de la communication ». En tant que telle, la compétence interculturelle requière l'acquisition de normes d'interaction qui se dégagent d'un examen détaillé de l'interaction verbale et non verbale mené à la fois sur sa propre langue culture et sur celle de l'interlocuteur.

En tentant de répondre précisément à cette question : « la compétence pragmatique peutelle être enseignée?», Kasper (1997) soutient le point de vue qu'il est possible de développer la compétence pragmatique dans le contexte d'activités pédagogiques planifiées. Selon une récapitulation d'investigations de pratiques de classes qui ont été conduites principalement au Japon et aux Etats-Unis, il est apparu de façon concluante que la compétence pragmatique des apprenants s'améliore quelle que soit l'approche 
pédagogique. Toutefois, les étudiants qui avaient bénéficié d'un enseignement explicite obtenaient des résultats meilleurs que ceux qui avaient acquis cette compétence de façon implicite. L'enseignement explicite implique généralement la description, l'explication, et la discussion des phénomènes pragmatiques des interactions. Cependant plusieurs obstacles à cette acquisition ont été mis en évidence.

- Les aspects socio-pragmatiques des interactions n'apparaissent généralement pas dans les discussions que présentent les méthodes actuelles d'apprentissage de langue (Liddicoat, 1997), qui continuent d'offrir des exercices et activités centrées sur la compétence linguistique (Steele \& Suozzo, 1994). Quand elles le font, c'est la perspective des apprenants qui est soulignée plutôt que celle de la communauté de la langue cible (Kramsch, 1987). Cette constatation suggère la nécessité de développer et de produire de nouvelles ressources pour l'enseignement de la langue, basée sur des recherches de type ethnographique qui mettent en avant les spécificités culturelles de la langue, et les aspects socio pragmatiques de la communication dans les différentes cultures (Liddicoat \& Crozet, 1997).

- Les professeurs de langue ont traditionnellement été formés à l'enseignement spécifique de la langue, qui adopte comme barre d'évaluation la compétence idéalisée du locuteur natif. Même lorsque les objectifs pédagogiques de l'apprentissage des langues se sont déplacés vers l'acquisition d'une compétence communicative, la majeur partie des échanges pratiqués dans la salle de classe ont continué de suivre un modèle répétitif. Le développement d'une meilleure compréhension de sa propre culture s'accompagne d'un savoir comprendre la culture cible qui ne signifie évidemment pas l'adoption aveugle de codes culturels étrangers, mais requière l'instauration d'une pratique d'auto-réflexion et d'engagement, c'est-à-dire une habileté à évaluer de façon critique sa propre culture et celle de l'autre sur la base de critères explicites, de perspectives et de pratiques multiples illustrées dans des ressources diversifiées (Gohard-Radenkovic, 1999).

- On continue de constater aussi que l'organisation des classes de langues, dominée par le professeur qui parle devant les élèves, ne favorise pas le développement de la compétence pragmatique, surtout lorsque l'on compare cette pratique aux conversations plus authentiques qui se tiennent en dehors de la salle de classe (Chaudron, 1998; MrowaHopkins, 1997). L'acquisition de la compétence interculturelle est limitée au nombre restreint des actes communicatifs qui sont échangés en classe.

- La communication para-verbale et le comportement non verbal sont encore moins présents dans les méthodes de langue, sauf peut-être lorsqu'il s'agit d'introduire un certain nombre de gestes emblématiques codifiés. Il est évident que ceci va à l'encontre du volume exceptionnel d'informations non verbales qui sont échangées dans les interactions quotidiennes.

Si l'on tient compte des observations précédentes, il devient essentiel pour les professeurs de langue d'intégrer les résultats des recherches sur les aspects sociopragmatiques de la communication dans leur pratique professionnelle. Dans la dernière section de cet article, nous présentons quelques échantillons d'activités basés sur notre corpus d'interactions qui, selon nous, devrait faire partie d'une approche pédagogique interculturelle.

\section{Échantillons d'activités}

Selon le niveau de compétence des apprenants dans la langue cible il existe plusieurs approches, mais toutes comprennent inévitablement une étape préalable d'observation 
pour faciliter la réflexion et la prise de conscience des spécificités culturelles dans sa propre culture et dans celle de l'autre avant de passer à des activités de repérage culturel et de développement de stratégies d'ajustement (Gohard-Radenkovic, 1999 : 144).

\section{Activités d'observation} amenée par l'observation d'interactions extraites de films contemporains, et l'identification de traits saillants dans des buts contrastifs et comparatifs. L'objectif est d'encourager l'apprenant à devenir soi-même chercheur indépendant, à observer des exemples semi authentiques d'interactions et à émettre des hypothèses sur les règles socioculturelles sous-jacentes tout en gardant l'esprit ouvert pour être prêt au besoin à modifier ses hypothèses initiales. Cette démarche contribue à réduire le risque de placarder une image stéréotypée sur son interlocuteur lorsqu'on se trouve soi-même en situation d'alloglotte avec des locuteurs de la langue cible (Balboni, 1999). certains comportements émotionnels, on donne aux apprenants une série de taches d'observations à l'intérieur et à l'extérieur de la classe pour déterminer, par exemple, si on peut dire avec certitude quand quelqu'un est fâché, dans quelles circonstances tel groupe se met en colère, et de quelle façon les interlocuteurs font l'expérience de la colère. Une tâche qui a été pratiquée avec succès dans un cours de français pour étudiants anglophones à l'université a consisté à soumettre un questionnaire à une dizaine de personnes de sa connaissance pour tester leurs réactions dans certaines situations. Par exemple, dans une de ces situations il est demandé "Comment réagissez-vous si quelqu'un passe devant vous alors que vous faites la queue depuis 10 minutes? ». On peut trouver d'autres exemples de situations de ce type sur un site internet du groupe Cultura au Massachusset Institute of Technology (Furstenberg et al, 2001) qui nous a inspiré pour cette activité. On peut aussi demander aux apprenants de regarder les scènes sélectionnées dans notre étude, et de répertorier les observations sur une grille d'analyse qui peut être partagée et commentée plus tard en classe. Le but de cette démarche est d'apprendre à décoder l'expression non verbale de la communication. Les critères d'observation pourraient être, par exemple, le niveau de familiarité entre les interactants, la circonstance précise qui provoque leur colère, et leur comportement non verbal quand ils observent les personnages en colère. Un exemple de cette grille que nous avons élaborée est présentée en annexe 2.

$\mathrm{Au}$ lieu d'utiliser une grille, on peut également imaginer des activités qui demandent aux apprenants de compléter ou de combiner des phrases, ou de sélectionner des réponses possibles dans une liste de scénarios. Il est possible aussi de demander de reconstituer des séquences correctes dans une conversation dans laquelle le comportement interactionnel des locuteurs natifs est conforme aux règles appropriées au contexte. Le but de ces activités est d'aider les apprenants à se concentrer sur les aspects para-verbaux et pragma-linguistiques de la communication. Les questions suivantes sont données à titre d'exemples :

- Dans cette conversation, quels mots montrent que les personnages sont en colère?

- Comment est-ce que l'intonation observée dans cette conversation diffère de celle qu'on peut observer dans des situations semblables dans votre propre langue? 
- Ce travail d'observation peut se faire en petits groupes, puis la discussion s'élargir au groupe classe dont on peut orienter l'attention à l'aide de questions telles que :

- Observe-t-on cette situation couramment dans votre culture? Dans quel contexte peut-elle se produire? Expliquez.

- Après avoir vu ces scènes, reconnaissez-vous une tendance culturelle? Décrivez-la.

- A quelle catégorie sociale peut-on associer cette tendance culturelle? Quelle valeur essentielle des Français, Italiens, Anglo-australiens illustre-t-elle?

- Dans une situation sociale, ou de travail (par exemple, un agent vous dit de ne pas vous garer dans une zone interdite au stationnement) à quoi pouvez-vous vous attendre?

\section{Application}

Les dernières questions mentionnées ci-dessus nous amènent à considérer des possibilités d'application du repérage culturel que les apprenants ont acquis au cours de la phase d'observation. Par exemple après avoir terminé les activités d'observations et de discussion en commun, on peut envisager de demander aux apprenants de prendre part à des jeux de rôle dans lesquels ils mettent en scène des comportements appropriés ou non à une situation donnée, et on peut demander à ceux qui ne participent pas d'émettre des hypothèses sur les réactions que pourraient provoquer ces scènes chez les membres du groupe culturel ciblé.

Une autre possibilité consiste à demander aux apprenants de rassembler des informations sur le contexte et les locuteurs (y compris l'antécédent de la colère, les rapports de pouvoir et de distance entre les personnages, ainsi que des informations se rapportant au contexte) à partir de scènes de films sélectionnées par l'enseignant qui montrent des exemples de personnages en colère. On demande ensuite aux apprenants d'écrire des scripts sur la base de ces informations pour un jeu de rôle. Après cela, on accorde un temps de préparation et les apprenants jouent leur scène que l'on filme en vidéo. L'enregistrement sera finalement passé en classe et les commentaires devront être orientés sur la justesse des comportements et les réactions possibles des membres de la culture cible. Finalement, on montre la scène originale du film pour la comparer et la contraster avec celle jouée par les apprenants.

On peut envisager d'autres façons d'impliquer les apprenants dans une réflexion sur les similarités et les différences de normes socioculturelles qui contrôlent l'expression émotionnelle, telle que celle qui consiste à demander aux apprenants d'écrire des entrées dans un journal où ils enregistrent leurs propres pensées à partir de leurs observations, ou bien d'échanger des questions avec des partenaires d'une autre école dans le pays d'origine ou celui de la langue cible, ou encore de composer un questionnaire ouvert afin de conduire une enquête ethnographique dans la communauté. Un exemple de cette activité peut se formuler ainsi : composez un questionnaire et engagez une conversation avec 10 personnes de votre connaissance, de milieux culturels et d'âge différents, sur les différences interculturelles, en utilisant quelques-unes des situations montrées dans les extraits de films comme entrée en matière. Le but de ces activités est de s'engager personnellement dans la culture de l'autre. Une fois que les apprenants commencent à développer une prise de conscience interculturelle de cette façon, ils sont prêts à développer la compétence pragmatique qui leur permettra de reconnaître et d'accepter la diversité culturelle, et de l'intégrer de façon enrichissante dans leur conscience personnelle. Certes la démarche succincte qui vient d'être esquissée mérite de plus 
longues élaborations qu'il ne nous est pas permis de développer dans le cadre du présent article.

\section{Conclusion}

Dans cet article, nous avons démontré comment intégrer la démarche et les résultats de notre recherche avec l'acquisition de la compétence interculturelle. Il ne fait pas de doute qu'une telle approche serait utile pour encourager les apprenants de langue à explorer les aspects socio-pragmatiques de la communication dans leur culture d'origine afin de parvenir à développer une connaissance méta-pragmatique. Le but ultime est d'éviter les catégorisations hâtives des comportements en termes de jugements de valeurs et dépasser le niveau des stéréotypes négatifs.

41 En ce qui concerne notre investigation, on ne peut tirer de conclusions définitives qui permettent de distinguer des différences culturelles frappantes entre les manifestations de colère déployées par les Anglo-australiens, les Français et les Italiens étant donné la quantité limitée de données collectées. Cependant, nos observations préliminaires indiquent des tendances intéressantes qui nous guideront dans la poursuite de nos analyses futures.

Essentiellement, ce projet nous a fourni l'occasion non seulement d'améliorer notre compréhension des normes contrôlant l'expression émotionnelle dans une perspective interculturelle, mais nous a permis aussi de partager nos idées avec les enseignants et les apprenants de langue. Nous pensons qu'il est essentiel de former à la fois les enseignants et les apprenants aux techniques d'observation et de recherche ethnographique, et de les encourager à découvrir les normes interactionnelles à travers l'analyse et l'explication systématique des variables culturelles qui se rapportent en particulier au contexte et aux relations interpersonnelles entre les groupes différenciés.

La manière la plus effective de former des enseignants et des apprenants de langue à distinguer les normes socioculturelles différenciées consiste à utiliser des exemples authentiques d'interaction dans une perspective contrastive, et à les encourager à mener leurs propres recherches. C'est, il nous semble, en suivant cette voie que nous pourrons admettre la diversité culturelle et développer notre sensibilité interculturelle, seule capable de limiter les généralisations et simplifications qui risquent de nous entraîner à l'application dangereuse de jugements stéréotypés.

BIBLIOGRAPHIE

Andersen, P. A., \& Guerrero, L. K. (1998). Principles of communication and emotion in social interaction. In P. A. Andersen, \& L. K. Guerrero (Eds.) Handbook of Communication and Emotion. San Diego : Academic Press. pp. 49-58. 
Archer, D., \& Akert, R. M. (1984). Problems of context and criterion in nonverbal communication : A new look at the accuracy issue. In M. Cook (Ed.) Issues in Person Perception. pp. 114-144. London: Methuen.

Balboni, P. E. (1999). Parole Comuni Culture Diverse : Guida Alla Comunicazione Interculturale. Marsilio.

Barraja-Rohan, A-M. (1999). Teaching conversation for intercultural competence. In J. Lo Bianco, A. J. Liddicoat \& C. Crozet (Eds.) Striving For the Third Place : Intercultural Competence through Language Education. pp. 143-153. Melbourne : Language Australia.

Bazzanella, C. (1994). Le Facce Del Parlare : Un Approccio Pragmatico All'italiano Parlato. Firenze : La Nuevo Italia.

Béal, C. (1990). "It's all in the asking" : A perspective on the problems of cross-cultural communication between native speakers of French and native speakers of Australian English in the workplace. Australian Review of Applied Linguistics, Series S, n 7. pp. 16-32.

Béal, C. (1992). "Did you have a good week-end ?" Or why there is no such thing as a simple question in cross-cultural encounters. Australian Review of Applied Linguistics, vol. 15, $\mathrm{n}^{\circ} 1$. pp. 23-52.

Birdwhistell, R. L. (1970). Kinesics and Context. Essay on Body Motion Communication. Philadelphia : University of Pennsylvania Press.

Burgeon, J. K., Buller, D.B., \& Woodall, W. G. (1989). Nonverbal Communication: The Unspoken Dialogue. New York : Haber and Row.

Byram, M. (1997). Teaching and Assessing Intercultural Communicative Competence. Clevedon : Multilingual Matters.

Carroli, P., Pavone, A., \& Tudini, V. (2003). Face value : Teaching Italian verbal and socio-cultural interaction. In Lo Bianco, J. \& Crozet, C., (Eds.) Teaching Invisible Culture : Classroom Practice and Theory. pp. 177-210. Melbourne : Language Australia.

Chaudron, C. (1988). Second Language Classrooms : Research on Teaching and Learning. Cambridge : Cambridge University Press.

Cosnier, J.\& Brossard, A. (1984). La Communication Non Verbale. Paris, Neuchâtel : Delachaux et Niestlé.

Ekman, P., \& Friesen, W. V. (1969). The repertoire of non verbal behaviour : origins, usage, codings. Semiotica, vol. 1. pp. 49-98.

Ekman, P., \& Friesen, W. V. (1971). Constants across cultures in the face and emotion. Jounal of Personality and Social Psychology, vol. 17. pp. 124-129.

Furstenberg, G., Levet, S., English, K., \& Maillet, K. (2001). Giving a virtual voice to the silent language of culture : The Cultura Project. In Language Learning \& Technology, vol. 5, n 1. pp. 55-102. Consulté le 20 septembre 2004: http://llt.msu.edu/vol5num1/furstenberg/default.html.

Gass, S.M. \& Varonis, E.M. (1991). Miscommunication in non-native speaker discourse. In N. Coupland, H. Giles \& J.M. Wiesmann (Eds.) Miscommunication and Problematic Talk. Newbury Park, CA : Sage.

Gohard-Radenkovic, A. (1999) Communiquer en langue étrangère. Berne: Peter Lang.

Gudykunst, W. B. \& Ting-Toomey, S. (1988). Culture and Interpersonal Communication. Beverly Hills: Sage Publication. 
Kasper, G. (1997). Can pragmatic competence be taught? University of Hawaï: Second Language Teaching and Curriculum Center. Consulté le 24 mars 2004 : http :/nflrc.hawaii.edu/NetWorks/ NW06/default.html.

Kramsch, C. (1987). The cultural discourse of foreign language textbooks. In A. J. Singer (Ed.) Toward A New Integration Of Language And Culture. Northeast conference on the teaching of foreign languages : Middlebury, V.T.

Liddicoat, A. J. (1997). Everyday speech as culture. In A.J. Liddicoat \& C. Crozet (Eds.) Teaching Language Teaching Culture. Australian Review of Applied Linguistics, Series S, 14. Australian National University. pp. 55-70.

Liddicoat, A.J. \& Crozet, C. (Eds.) (1997). Teaching Language Teaching Culture. Australian Review of Applied Linguistics, Series S, 14. Australian National University.

Markus, H., \& Kitayama, S. (1994). The cultural construction of self and emotion : Implications for social behavior. In S. Kitayama, \& H. Markus, (Eds.), Culture And Emotion. pp. 89-130. American Psychological Association.

Matsumoto, D., Kudoh, T., Scherer, K. R., \& Wallbott, H. G. (1988). Antecedents of and reactions to emotions in the US and Japan. Journal of Cross-Cultural Psychology, vol. 11. pp. 551-555.

Matsumoto, D., H.G. Wallbott, \& K. R. Scherer (1994). Emotions in intercultural communication. In M. Asante \& W. Gudykunst (Eds.), Handbook of intercultural and international communication. Beverly Hills : Sage. pp. 225-246.

Mehrabian, A. (1969). Significance of posture and position in the communication of attitude and status relationships. Psychological Bulletin, vol. 71. pp. 359-72.

Mehrabian, A. (1972). Nonverbal Communication. Chicago : Aldine.

Mrowa-Hopkins, C. (1997). Communication, Discourse, Interaction in Language Classes. Thèse de doctorat, University of Adelaide, Adelaide, South Australia.

Peeters, B. (2003). Le transculturel : sémantique, pragmatique, axiologie. La Linguistique, vol. 39, n 1. pp. 119-133.

Porter, R. E. \& Samovar, L. A. (1998). Cultural influences on emotional expression: Implications for intercultural communication. In Handbook of Communication and Emotion, P.A. Andersen \& L. K. Guerrero (Eds.). pp. 451-470.

Rimé, B., Corsini, S. \& Herbette, G. (2002). Emotion, verbal expression, and the social sharing of emotion. In The Verbal Communication of Emotions : Interdisciplinary Perspectives, S. R. Fussell (Ed.) London : Lawrence Erlbaum.

Russell, J. A. et al (Eds.) (1995). Everyday Conceptions of Emotion. Netherlands : Kluwer Academic Press.

Scherer, K. R. (1986). Vocal affect expression : A review and a model for future research. Psychological Bulletin, vol. 99. pp. 143-165.

Scherer, K. R., \& Ekman, P. (1982). Handbook of Methods in Non-Verbal Behavior Research. Cambridge : Cambridge University Press.

Scherer, K. R., \& Wallbott, H. G. (1994). Evidence for universality and cultural variation of differential emotion response patterning. Journal of Personality and Social Psychology, vol. $66, \mathrm{n}^{\circ} 2$. pp. 310-328.

Steele, R. \& Suozzo, A. (1994). Teaching French Culture: Theory and Practice. Illinois, US : National Textbook Company. 
Vion, R. (1992). La Communication Verbale: Analyse des Interactions. Paris : Hachette.

Wierzbicka, A (1999). Emotions Across Languages And Cultures - Diversity And Universals. Cambridge: Cambridge University Press.

\section{ANNEXES}

\section{Annexe 1 : Sélection de films long métrage}

\section{Films australiens:}

The Dish, 2000, réal. Rob Sitch.

Mullet, 2001, réal. David Caesar.

Go Big, 2003, réal. Tony Tilse.

He Died With A Felafel In His Hand, 2001, réal. Richard Lowenstein.

Lantana, 2002, réal. Ray Lawrence.

Secret Mens' Business, 1999, réal. Ken Cameron.

Films français :

Va Savoir (Who knows ?), 2002, réal. Jacques Rivette.

Le Chaos (Chaos), 2003, réal. Coline Serreau.

Sur mes lèvres (Read my lips), 2001, réal. Jacques Audiard.

Le gout des autres (It takes all kinds / The taste of others), 2000, réal. Agnès Jaoui.

Le placard (The closet), 2001, réal. Francis Veber.

Grégroire Moulin contre l'humanité (G M against humanity), 2001, dir. Artus De Penguern.

Une affaire privée (Private investigation), 2002, réal. Guillaume Nicloux.

\section{Films italiens :}

Commissario Montalbano - L'odore della Notte (Inspector Montalbano - The scent of the night), 2002, réal. Alberto Sironi.

Domani (Tomorrow), 2001, réal. Francesca Archibugi

Giorni (Days), 2001, réal. Laura Muscardin.

L'ultimo bacio (Last Kiss), 2000, réal. Gabriele Muccino.

Pane e tulipani (Bread and Tulips), 2000, réal. Silvio Soldini.

Per tutto il tempo che ci resta (Acts of Justice), 1998, réal. Vincenzo Terracciano.

Tre storie (Three stories), 1999, réal. Piergiorgio Gay \& Roberto San Pietro. 


\section{Annexe 2: Grille d'analyse}

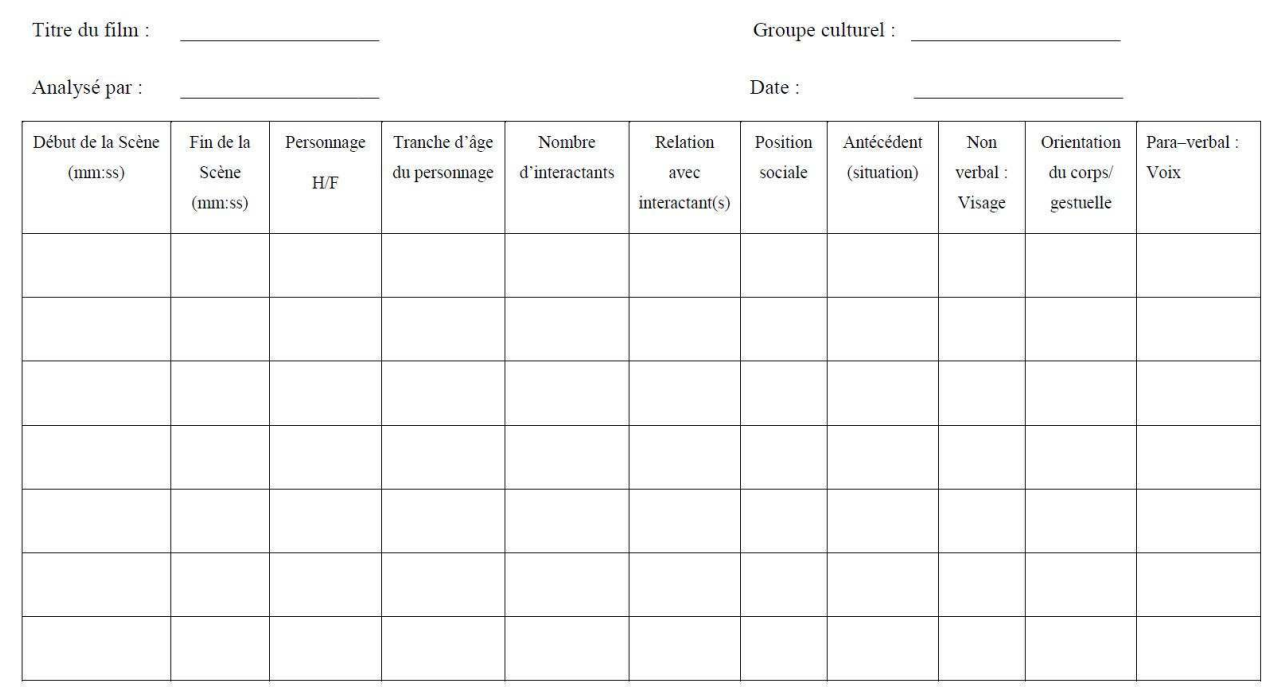

Annexe 3 : Le Coeur : Scene $2(00: 09: 10)$

\section{Scenario :}

Location : de jour, bureau sans cloison qui ouvre sur un couloir, murs couleur vives.

Rôle des participants : JEFF, le patron, et sa secrétaire SECR + un collègue de Jeff, ALEX

Déplacements : les personnages sont debout se faisant face dans le couloir; Jeff est sur le point de partir, il enfile sa veste lorsque SECR lui rappelle son rendez-vous.

Propos : échange d'information

Émotions et déroulement de l'interaction : SECR dévoile son irritation (L.7) en réponse aux questions de son patron. La réaction de Jeff (L.8) contribue à accentuer l'irritation qui se traduit par de la familiarité (L.9) pouvant entrainer des conséquences négatives dans les relations hiérarchiques. Les rapports de pouvoir sont déstabilisés quand Jeff réagit de façon infantile (mimique le ton de sa secrétaire), ceci a un effet comique qui diffuse la tension. JEFF met fin à la scène par une remarque (stratégie d'analyse) adressée à un tiers.

Script culturel : la règle sociale qui demande qu'une secrétaire s'adresse à son patron avec respect est contournée. La secrétaire adopte un ton de familiarité. Jeff reconnait ce dérèglement et tente de justifier cette rupture dans les relations sociales auprès d'un tiers en invoquant un contexte politique de gauche.

\section{Transcript :}




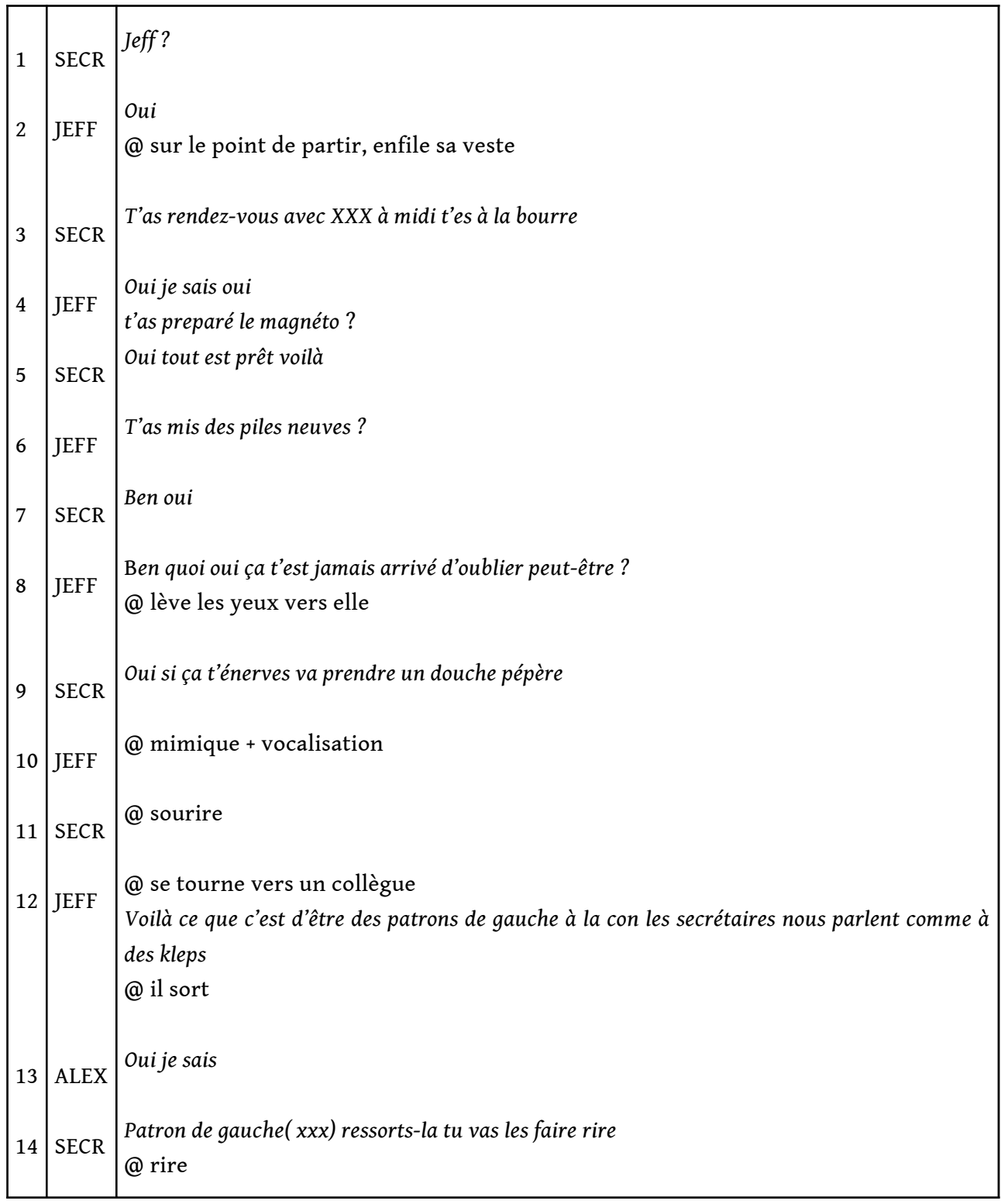

\section{NOTES}

1. Voir en particulier: Russell et al, 1995 ; Andersen \& Guerrero, 1998 ; Rimé et al., 2002.

2. Elles correspondent à ce que Ekman et Freisen (1971) nomment « Social rules ».

3. Notre traduction de l'expression "being nice".

4. Un exemple extrait du film français Le Coeur des Hommes a été choisi à titre illustratif. Il n'est pas possible dans le cadre de cet article, dont le but est de démontrer un application pédagogique, de donner une analyse plus complète des résultats de notre analyse comparative. Cette analyse fera l'objet d'une publication ultérieure. 


\section{RÉSUMÉS}

Notre étude s'inscrit dans une perspective à la fois interrelationnelle et interculturelle et porte sur la dimension émotionnelle de la communication entre locuteurs de groupes culturellement différenciés. La démarche que nous avons adoptée s'articule autour d'une réflexion théorique plus large portant sur le comportement non verbal dans l'expression et la communication des émotions dans les relations interpersonnelles impliquant divers degrés d'investissement de soi et de distanciation. Notre attention se portera en particulier sur les manifestations de la colère, et les différences observées entre les trois groupes culturels sélectionnés dans notre étude.

\section{INDEX}

Mots-clés : interculturel, non verbal, émotions, colère

\section{AUTEURS}

\section{COLETTE MROWA-HOPKINS}

Méridionale, section de Français, université Flinders, Adélaïde, Australie Colette Mrowa-Hopkins enseigne le français dans le Département de Langues à l'Université Flinders en Australie du Sud. Son domaine de recherches comprend la didactique des langues, les TIC, l'acquisition de la langue seconde, la communication interculturelle, et les médias Elle dirige également avec Antonella Strambi un cours de formation pédagogique pour enseignants de langue.

Courriel : colette.mrowa-hopkins[at]flinders.edu.au.

\section{ANTONELLA STRAMBI}

Méridionale, section d'Italien, université Flinders, Adélaïde, Australie

Antonella Strambi enseigne l'italien dans le Département de Langues à l'Université Flinders en Australie du Sud. Son domaine de recherches comprend la didactique des langues, les TIC, l'acquisition de la langue seconde, la communication interculturelle, et les médias. Elle dirige également avec Colette Mrowa-Hopkins un cours de formation pédagogique pour enseignants de langue.

Courriel : antonella.strambi[at]flinders.edu.au. 